\title{
Synthesis of Leap-Frog Multiple-Loop Feedback OTA-C Filters
}

\author{
Yichuang Sun, Senior Member, IEEE
}

\begin{abstract}
Operational transconductance amplifier and capacitor filters based on the leap-frog (LF) structure are studied from the viewpoint of multiple-loop feedback (MLF) and coefficient matching. The LF configuration is obtained from an MLF model, which has the minimum number of components and only grounded capacitors. Explicit and iterative design formulas are derived for the synthesis of arbitrary filter characteristics. All-pole filters are realized using the basic LF structure, and filters with arbitrary transmission zeros are synthesized by adding the input distributor or output summer.
\end{abstract}

Index Terms-Active filters, analog circuits, continuous-time filters, operational transconductance amplifier and capacitor (OTA-C) filters.

\section{INTRODUCTION}

$\mathbf{T}$ HE leap-frog (LF) structure is one of the most popular choices in active- $R C$ filter design [1], [2] due to its lower sensitivity than the cascade method. LF operational transconductance amplifier and capacitor (OTA-C) filters have been derived by simulating passive $R L C$ ladder prototypes [2]-[5] and using a systematic multiple-loop feedback (MLF) approach [2], [6], [7]. The difference between the LF OTA-C filter structures based on the two approaches is that circuit component values are obtained from simulated $R L C \mathrm{~s}$ in the former and determined by coefficient matching equations in the latter. Active filter design based on the $R L C$ ladder simulation approach needs knowledge of passive $R L C$ filters and treatment of input and output terminated impedances, while the MLF approach does not have these problems. More important is that the LF MLF approach can realize any transmission zeros, whilst the LF simulation method can only realize imaginary zeros. One of the most important applications of OTA-C filters is the linear phase filtering in computer hard-disk-drive systems. In this application, real zeros are normally required, which may only be realized by the MLF approach when using the LF structure. In communication receivers and video systems, equalizers with certain transmission zeros are often required for stringent phase requirements, for which the LF simulation approach may not be suitable.

Compared with other MLF configurations, such as the follow-the-leader feedback (FLF) and inverse-follow-theleader-feedback (IFLF) structures that have similar sensitivity, the LF MLF configuration has the minimum sensitivity [1], [2]. Unlike the FLF and similar to IFLF, it does not have a multiple feedback input summation node, which may simplify the IC implementation in practice because the summing node usually

Manuscript received April 6, 2005; revised August 3, 2005, and November 4, 2005. This paper was recommended by Associate Editor A. Korotkov.

The author is with the School of Electronic, Communication and Electrical Engineering, University of Hertfordshire, Hatfield Herts AL10 9AB, U.K. (e-mail: y.sun@herts.ac.uk).

Digital Object Identifier 10.1109/TCSII.2006.879095 has high-frequency parasitic effects. When the OTA parasitics are considered, in particular, the input capacitance in the differential applications, the FLF and IFLF configurations may suffer more serious performance degradation because of long feedback paths from the $n$th node to the input node. Because each path is capacitive coupling, the effects on circuit poles and zeros appear at high frequencies. Obviously, the extent of those effects can be considered as proportional to the ratio of parasitic capacitance to circuit integrating capacitance. The LF structure is also advantageous over the cascade configuration [1], [2]. Recent investigations have demonstrated that LF MLF OTA-C filters offer better passband magnitude sensitivity, maximum input voltage, and magnitude frequency response considering OTA nonideality effects over IFLF and cascade OTA-C filters [8]. In particular, the noise performance of the LF filters has been shown to be much better than that of the IFLF filters. Furthermore, promising results from recent research of use of the LF MLF OTA-C filters for read channel applications show that the LF MLF design method could be an attractive alternative to other methods [9]. However, the synthesis of the LF MLF structure is not as simple as the FLF and IFLF structures. While simple explicit formulas exist for the FLF [10], [11] and IFLF [12], [13] configurations that can be easily used [14], an iterative process is required for LF synthesis. As well as general iterative equations, straightforward formulas for the most often used orders of filters should be derived for ready use.

The synthesis of LF OTA-C filters for realizations of both transmission poles and zeros can be conducted using the general MLF approach [2], [6], [7]. This synthesis method, however, is based on matrix manipulation and requires knowledge of the general MLF theory. In this paper, we present an alternative iterative approach for synthesis of LF MLF OTA-C filters based on coefficient matching.

\section{All-Pole LF FEEDBACK OTA-C FILTERS}

\section{A. Formulation of Circuit Transfer Function}

The general all-pole LF OTA-C filter configuration [2], [6] is depicted in Fig. 1. It has the minimum number of components and uses only grounded capacitors. With time constant $\tau_{j}=$ $C_{j} / g_{j}$, we can write the equations of the circuit as

$$
\begin{aligned}
& V_{o 1}=\left(V_{i 1}-V_{o 2}\right) /\left(\tau_{1} s\right) \\
& V_{o j}=\left(V_{o(j-1)}+V_{i j}-V_{o(j+1)}\right) /\left(\tau_{j} s\right) \\
& V_{\text {on }}=\left(V_{o(n-1)}+V_{\text {in }}-V_{\text {on }}\right) /\left(\tau_{n} s\right), \quad j=2,3, \ldots, n-1
\end{aligned}
$$

where $s$ is the complex frequency, $V_{o j}$ is the output voltage of the $j$ th integrator, and $V_{i j}$ can be imagined as a voltage source inserted between the output $\left(V_{o(j-1)}\right)$ of the $(j-1)$ th integrator and the noninverting terminal of the $j$ th OTA. Note that $V_{i 1}=V_{\text {input }}, V_{\text {on }}=V_{\text {output }}$ and $\left(V_{o(j-1)}+V_{i j}\right)$ can be viewed 


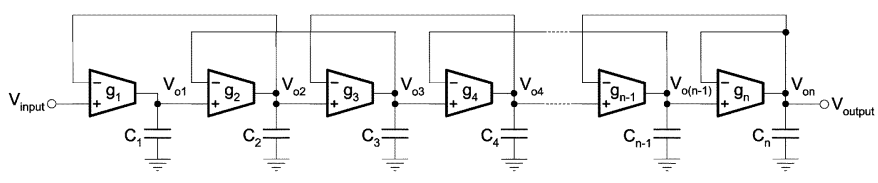

Fig. 1. General all-pole LF configuration.

equivalently as the input voltage (to the noninverting terminal) of the $j$ th OTA. Introducing $V_{i j}$ is mainly for the convenience of defining $G$ functions below and facilitating derivations in Section III. For noise analysis, $V_{i j}$ are input-referral noise sources and $G$ functions are the intermediate functions [2], [8].

Defining the transfer function from the filter input to the output of integrator $j$ as

$H_{j}(s)=V_{o j} / V_{i 1}=N_{H j}(s) / D(s), \quad j=1,2,3, \ldots, n$

it can be shown from (1) that

$$
\begin{aligned}
N_{H n}(s)= & 1 \\
N_{H(n-1)}(s)= & \tau_{n} s+1 \\
N_{H j}(s)= & \tau_{j+1} s N_{H(j+1)}(s)+N_{H(j+2)}(s), \\
& j=n-2, n-3, \ldots, 1 \\
D(s)= & \tau_{1} s N_{H 1}(s)+N_{H 2}(s) .
\end{aligned}
$$

Note that (3) and (4) show that $N_{H j}(s)$ and $D(s)$ can be obtained in an iterative way.

Defining the transfer function from the input of integrator $j$ to the filter output as

$G_{j}(s)=V_{\text {on }} / V_{i j}=N_{G j}(s) / D(s), \quad j=1,2,3, \ldots, n$

from (1) we can obtain

$$
\begin{aligned}
N_{G 1}(s) & =1 \\
N_{G 2}(s)= & \tau_{1} s \\
N_{G j}(s)= & \tau_{j-1} s N_{G(j-1)}(s)+N_{G(j-2)}(s), \\
& \quad j=3,4, \ldots, n \\
D(s)= & \left(\tau_{n} s+1\right) N_{G n}(s)+N_{G(n-1)}(s) .
\end{aligned}
$$

Again, note that $N_{G j}(s)$ and $D(s)$ in (6) and (7) can be determined iteratively.

It can be shown that the $H$ and $G$ functions have the following relation:

$$
\begin{gathered}
D(s)=N_{G j}(s) N_{H(j-1)}(s)+N_{G(j-1)}(s) N_{H j}(s), \\
j=2,3, \ldots, n .
\end{gathered}
$$

For any order, using the iterative formulas in (3), we can obtain all associated numerator polynomials of the $H$ functions. For order $n=3,4,5$, and 6 , it can be shown that

$$
\begin{aligned}
n & =3: N_{H 3}(s)=1 \\
N_{H 2}(s) & =\tau_{3} s+1 \\
N_{H 1}(s) & =\tau_{2} \tau_{3} s^{2}+\tau_{2} s+1
\end{aligned}
$$

$$
\begin{aligned}
n= & 4: N_{H 4}(s)=1 \\
N_{H 3}(s)= & \tau_{4} s+1 \\
N_{H 2}(s)= & \tau_{3} \tau_{4} s^{2}+\tau_{3} s+1 \\
N_{H 1}(s)= & \tau_{2} \tau_{3} \tau_{4} s^{3}+\tau_{2} \tau_{3} s^{2}+\left(\tau_{2}+\tau_{4}\right) s+1 \\
n= & 5: N_{H 5}(s)=1 \\
N_{H 4}(s)= & \tau_{5} s+1 \\
N_{H 3}(s)= & \tau_{4} \tau_{5} s^{2}+\tau_{4} s+1 \\
N_{H 2}(s)= & \tau_{3} \tau_{4} \tau_{5} s^{3}+\tau_{3} \tau_{4} s^{2}+\left(\tau_{3}+\tau_{5}\right) s+1 \\
N_{H 1}(s)= & \tau_{2} \tau_{3} \tau_{4} \tau_{5} s^{4}+\tau_{2} \tau_{3} \tau_{4} s^{3}+\left[\tau_{2}\left(\tau_{3}+\tau_{5}\right)+\tau_{4} \tau_{5}\right] s^{2} \\
& +\left(\tau_{2}+\tau_{4}\right) s+1 \\
n= & 6: N_{H 6}(s)=1 \\
N_{H 5}(s)= & \tau_{6} s+1 \\
N_{H 4}(s)= & \tau_{5} \tau_{6} s^{2}+\tau_{5} s+1 \\
N_{H 3}(s)= & \tau_{4} \tau_{5} \tau_{6} s^{3}+\tau_{4} \tau_{5} s^{2}+\left(\tau_{4}+\tau_{6}\right) s+1 \\
N_{H 2}(s)= & \tau_{3} \tau_{4} \tau_{5} \tau_{6} s^{4}+\tau_{3} \tau_{4} \tau_{5} s^{3}+\left[\tau_{3}\left(\tau_{4}+\tau_{6}\right)+\tau_{5} \tau_{6}\right] s^{2} \\
& +\left(\tau_{3}+\tau_{5}\right) s+1 \\
N_{H 1}(s)= & \tau_{2} \tau_{3} \tau_{4} \tau_{5} \tau_{6} s^{5}+\tau_{2} \tau_{3} \tau_{4} \tau_{5} s^{4} \\
& +\left\{\left[\tau_{3}\left(\tau_{4}+\tau_{6}\right)+\tau_{5} \tau_{6}\right] \tau_{2}+\tau_{4} \tau_{5} \tau_{6}\right\} s^{2} \\
& +\left[\tau_{2}\left(\tau_{3}+\tau_{5}\right)+\tau_{4} \tau_{5}\right] s^{2} \\
& +\left(\tau_{2}+\tau_{4}+\tau_{6}\right) s+1 .
\end{aligned}
$$

Also, using the iterative formulas in (6), we can obtain corresponding $N_{G j}(s)$ for order $n=3,4,5$, and 6 , given as

$$
\begin{aligned}
n= & 3: N_{G 1}(s)=1 \\
N_{G 2}(s)= & \tau_{1} s \\
N_{G 3}(s)= & \tau_{1} \tau_{2} s^{2}+1 \\
n= & 4: N_{G 1}(s)=1 \\
N_{G 2}(s)= & \tau_{1} s \\
N_{G 3}(s)= & \tau_{1} \tau_{2} s^{2}+1 \\
N_{G 4}(s)= & \tau_{1} \tau_{2} \tau_{3} s^{3}+\left(\tau_{1}+\tau_{3}\right) s \\
n= & 5: N_{G 1}(s)=1 \\
N_{G 2}(s)= & \tau_{1} s \\
N_{G 3}(s)= & \tau_{1} \tau_{2} s^{2}+1 \\
N_{G 4}(s)= & \tau_{1} \tau_{2} \tau_{3} s^{3}+\left(\tau_{1}+\tau_{3}\right) s \\
N_{G 5}(s)= & \tau_{1} \tau_{2} \tau_{3} \tau_{4} s^{4}+\left[\tau_{1} \tau_{2}+\left(\tau_{1}+\tau_{3}\right) \tau_{4}\right] s^{2}+1 \\
\mathrm{n}= & 6: N_{G 1}(s)=1 \\
N_{G 2}(s)= & \tau_{1} s \\
N_{G 3}(s)= & \tau_{1} \tau_{2} s^{2}+1 \\
N_{G 4}(s)= & \tau_{1} \tau_{2} \tau_{3} s^{3}+\left(\tau_{1}+\tau_{3}\right) s \\
N_{G 5}(s)= & \tau_{1} \tau_{2} \tau_{3} \tau_{4} s^{4}+\left[\tau_{1} \tau_{2}+\left(\tau_{1}+\tau_{3}\right) \tau_{4}\right] s^{2}+1 \\
N_{G 6}(s)= & \tau_{1} \tau_{2} \tau_{3} \tau_{4} \tau_{5} s^{5} \\
& +\left\{\tau_{1} \tau_{2} \tau_{3}+\left[\tau_{1} \tau_{2}+\left(\tau_{1}+\tau_{3}\right) \tau_{4}\right] \tau_{5}\right\} s^{3} \\
& +\left(\tau_{1}+\tau_{3}+\tau_{5}\right) s .
\end{aligned}
$$

Note that the formulated numerator polynomials of the respective $H$ and $G$ functions are useful for filter sensitivity, maximum input voltage, and noise analysis [8]. 
If $V_{i 1}$ is taken as the overall input and $V_{\text {on }}$ as the overall output, the overall transfer function $H(s)$ can be obtained using either the $H$ or $G$ functions, given by

$$
H(s)=V_{\text {output }} / V_{\text {input }}=H_{n}(s)=G_{1}(s)=1 / D(s) .
$$

Using (4) and (7) and corresponding $H$ and $G$ functions, one can obtain the denominator polynomial $D(s)$ of respective overall transfer functions as

$$
\begin{aligned}
n= & 3: D(s)=\tau_{1} \tau_{2} \tau_{3} s^{3}+\tau_{1} \tau_{2} s^{2}+\left(\tau_{1}+\tau_{3}\right) s+1 \\
n= & 4: D(s)=\tau_{1} \tau_{2} \tau_{3} \tau_{4} s^{4}+\tau_{1} \tau_{2} \tau_{3} s^{3} \\
& +\left(\tau_{1} \tau_{2}+\tau_{1} \tau_{4}+\tau_{3} \tau_{4}\right) s^{2}+\left(\tau_{1}+\tau_{3}\right) s+1 \\
n= & 5: D(s)=\tau_{1} \tau_{2} \tau_{3} \tau_{4} \tau_{5} s^{5}+\tau_{1} \tau_{2} \tau_{3} \tau_{4} s^{4} \\
& +\left(\tau_{1} \tau_{2} \tau_{3}+\tau_{1} \tau_{2} \tau_{5}+\tau_{1} \tau_{4} \tau_{5}+\tau_{3} \tau_{4} \tau_{5}\right) s^{3} \\
& +\left(\tau_{1} \tau_{2}+\tau_{1} \tau_{4}+\tau_{3} \tau_{4}\right) s^{2}+\left(\tau_{1}+\tau_{3}+\tau_{5}\right) s+1 \\
n= & 6: D(s)=\tau_{1} \tau_{2} \tau_{3} \tau_{4} \tau_{5} \tau_{6} s^{6}+\tau_{1} \tau_{2} \tau_{3} \tau_{4} \tau_{5} s^{5} \\
& +\left(\tau_{1} \tau_{2} \tau_{3} \tau_{4}+\tau_{1} \tau_{2} \tau_{3} \tau_{6}\right. \\
& \left.+\tau_{1} \tau_{2} \tau_{5} \tau_{6}+\tau_{1} \tau_{4} \tau_{5} \tau_{6}+\tau_{3} \tau_{4} \tau_{5} \tau_{6}\right) s^{4} \\
+ & \left(\tau_{1} \tau_{2} \tau_{3}+\tau_{1} \tau_{2} \tau_{5}+\tau_{1} \tau_{4} \tau_{5}+\tau_{3} \tau_{4} \tau_{5}\right) s^{3} \\
& +\left(\tau_{1} \tau_{2}+\tau_{1} \tau_{4}+\tau_{1} \tau_{6}+\tau_{3} \tau_{4}+\tau_{3} \tau_{6}\right. \\
& \left.+\tau_{5} \tau_{6}\right) s^{2}+\left(\tau_{1}+\tau_{3}+\tau_{5}\right) s+1 . \\
&
\end{aligned}
$$

\section{B. Formulas for Determination of Parameter Values}

To realize the general unity gain all-pole transfer function

$$
H_{d}(s)=1 /\left(B_{n} s^{n}+B_{n-1} s^{n-1}+\cdots+B_{1} s+1\right)
$$

the explicit expressions for determining parameter values can be obtained from the coefficient matching equations based on the comparison between the coefficients of $H(s)$ and those in (22). The following are the derived design formulas:

$$
\begin{aligned}
n & =3: \tau_{3}=B_{3} / B_{2} \\
\tau_{2} & =B_{2} /\left(B_{1}-\tau_{3}\right) \\
\tau_{1} & =B_{1}-\tau_{3} \\
n & =4: \tau_{4}=\frac{B_{4}}{B_{3}} \\
\tau_{3} & =\frac{B_{3}}{B_{2}-B_{1} \tau_{4}} \\
\tau_{2} & =\frac{B_{2}-B_{1} \tau_{4}}{B_{1}-\tau_{3}} \\
\tau_{1} & =B_{1}-\tau_{3} \\
n & =5: \tau_{5}=\frac{B_{5}}{B_{4}} \\
\tau_{4} & =\frac{B_{4}}{B_{3}-B_{2} \tau_{5}} \\
\tau_{3} & =\frac{B_{3}-B_{2} \tau_{5}}{B_{2}-\left(B_{1}-\tau_{5}\right) \tau_{4}} \\
\tau_{2} & =\frac{B_{2}-\left(B_{1}-\tau_{5}\right) \tau_{4}}{B_{1}-\tau_{3}-\tau_{5}} \\
\tau_{1} & =B_{1}-\tau_{3}-\tau_{5} \\
n & =6: \tau_{6}=\frac{B_{6}}{B_{5}} \\
\tau_{5} & =\frac{B_{5}}{B_{4}-B_{3} \tau_{6}}
\end{aligned}
$$

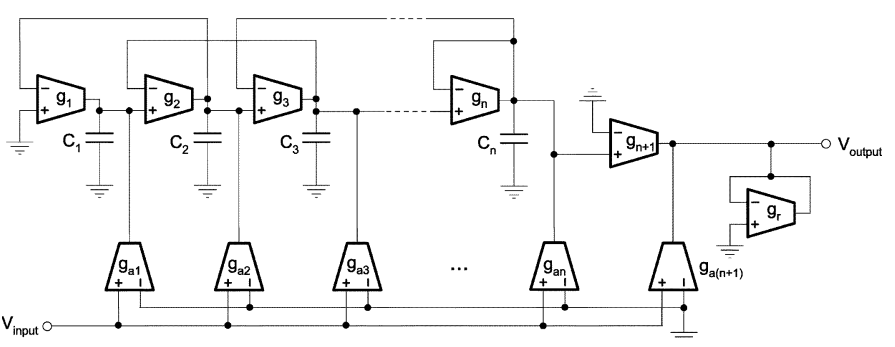

Fig. 2. Universal LF structure with input distribution OTA network.

$$
\begin{aligned}
\tau_{4} & =\frac{B_{4}-B_{3} \tau_{6}}{B_{3}-\left(B_{2}-B_{1} \tau_{6}\right) \tau_{5}} \\
\tau_{3} & =\frac{B_{3}-\left(B_{2}-B_{1} \tau_{6}\right) \tau_{5}}{B_{2}-\left(B_{1}-\tau_{5}\right) \tau_{4}-B_{1} \tau_{6}} \\
\tau_{2} & =\frac{B_{2}-\left(B_{1}-\tau_{5}\right) \tau_{4}-B_{1} \tau_{6}}{B_{1}-\tau_{3}-\tau_{5}} \\
\tau_{1} & =B_{1}-\tau_{3}-\tau_{5} .
\end{aligned}
$$

\section{LF MLF OTA-C FILTERS WITH TRANSMISSION ZEROS}

\section{A. Functions and Formulas for Input Distributor Type}

By adding the input distribution OTA network to the all-pole LF structure in Fig. 1 to produce zeros, the desired general transfer function in (27) can be realized:

$$
H_{d}(s)=\frac{A_{n} s^{n}+A_{n-1} s^{n-1}+\cdots+A_{1} s+A_{0}}{B_{n} s^{n}+B_{n-1} s^{n-1}+\cdots+B_{1} s+1} .
$$

The resultant general structure is shown in Fig. 2 .

Denoting $\beta_{j}=g_{a j} / g_{j}, \gamma=g_{n+1} / g_{r}$, and $\beta_{n+1}=g_{a(n+1)} / g_{n+1}$, the circuit equations can be written from Fig. 2 as

$$
\begin{aligned}
\tau_{1} s V_{o 1} & =-V_{o 2}+\beta_{1} V_{\text {input }}, \quad j=2,3,4, \ldots, n-1 \\
\tau_{j} s V_{o j} & =V_{o(j-1)}-V_{o(j+1)}+\beta_{j} V_{\text {input }} \\
\tau_{n} s V_{\text {on }} & =V_{o(n-1)}-V_{\text {on }}+\beta_{n} V_{\text {input }} \\
V_{\text {output }} & =\gamma\left(V_{\text {on }}+\beta_{n+1} V_{\text {input }}\right)
\end{aligned}
$$

where, equivalent to (1), we have $V_{i j}=\beta_{j} V_{\text {input }}$.

The set of equations in (28) can be rearranged with $H_{j}(s)=$ $V_{o j} / V_{\text {input }}$ and $N_{G j}(s)$ as

$$
\begin{aligned}
N_{G 2}(s) H_{1}(s)= & -H_{2}(s)+\beta_{1} \\
N_{G(h+1)}(s) H_{h}(s)= & -N_{G h}(s) H_{h+1}(s)+\sum_{j=1}^{h} \beta_{j} N_{G j}(s), \\
& \quad h=2,3, \ldots, n-1 \\
\left(\tau_{n} s+1\right) N_{G n}(s) H_{n}(s)= & -N_{G(n-1)}(s) H_{n}(s)+\sum_{j=1}^{n} \beta_{j} N_{G j}(s) .
\end{aligned}
$$

From the last equation in (29), we can solve for $H_{n}(s)$ to obtain

$$
\begin{aligned}
H_{n}(s) & =\frac{V_{\text {on }}}{V_{\text {input }}} \\
& =\frac{1}{\left(\tau_{n} s+1\right) N_{G n}(s)+N_{G(n-1)}(s)} \sum_{j=1}^{n} \beta_{j} N_{G j}(s) .
\end{aligned}
$$


The expression of the overall transfer function is obtained from the last relation in (28) as

$$
H(s)=V_{\text {output }} / V_{\text {input }}=\gamma\left(\beta_{n+1}+H_{n}(s)\right) .
$$

By substituting (30) into (31), we obtain the transfer function as

$$
H(s)=\gamma\left[\beta_{n+1}+\frac{\sum_{j=1}^{n} \beta_{j} N_{G j}(s)}{\left(\tau_{n} s+1\right) N_{G n}(s)+N_{G(n-1)}(s)}\right]
$$

where $N_{G j}(s)$ can be obtained from (6) iteratively and those for $n=3-6$ have been given in Section II-A.

Using (6) and (32), we can derive the numerators $N(s)$ of the transfer functions and the corresponding design formulas to determine the distribution parameters $\beta_{j}$ when realizing the general function in (27) for $n=3-6$ as

$$
\begin{aligned}
& n=3: N(s)=\beta_{4} \tau_{1} \tau_{2} \tau_{3} s^{3}+\left(\beta_{4}+\beta_{3}\right) \tau_{1} \tau_{2} s^{2} \\
& +\left[\beta_{4}\left(\tau_{1}+\tau_{3}\right)+\beta_{2} \tau_{1}\right] s+\left(\beta_{4}+\beta_{3}+\beta_{1}\right) \\
& \beta_{4}=A_{3} / B_{3} \\
& \beta_{3}=A_{2} / B_{2}-\beta_{4} \\
& \beta_{2}=\left(A_{1}-\beta_{4} B_{1}\right) / \tau_{1} \\
& \beta_{1}=A_{0}-\left(\beta_{4}+\beta_{3}\right) \\
& n=4: N(s)=\beta_{5} \tau_{1} \tau_{2} \tau_{3} \tau_{4} s^{4}+\left(\beta_{5}+\beta_{4}\right) \tau_{1} \tau_{2} \tau_{3} s^{3} \\
& +\left[\beta_{5}\left(\tau_{1} \tau_{2}+\tau_{1} \tau_{4}+\tau_{3} \tau_{4}\right)+\beta_{3} \tau_{1} \tau_{2}\right] s^{2} \\
& +\left[\left(\beta_{5}+\beta_{4}\right)\left(\tau_{1}+\tau_{3}\right)+\beta_{2} \tau_{1}\right] s \\
& +\left(\beta_{5}+\beta_{3}+\beta_{1}\right) \\
& \beta_{5}=A_{4} / B_{4} \\
& \beta_{4}=A_{3} / B_{3}-\beta_{5} \text {, } \\
& \beta_{3}=\left(A_{2}-\beta_{5} B_{2}\right) / \tau_{1} \tau_{2} \\
& \beta_{2}=\left[A_{1}-\beta_{5} B_{1}-\beta_{4}\left(\tau_{1}+\tau_{3}\right)\right] / \tau_{1} \\
& \beta_{1}=A_{0}-\left(\beta_{5}+\beta_{3}\right) \\
& n=5: N(s)=\beta_{6} \tau_{1} \tau_{2} \tau_{3} \tau_{4} \tau_{5} s^{5} \\
& +\left(\beta_{6}+\beta_{5}\right) \tau_{1} \tau_{2} \tau_{3} \tau_{4} s^{4} \\
& +\left[\beta_{6}\left(\tau_{1} \tau_{2} \tau_{3}+\tau_{1} \tau_{2} \tau_{5}+\tau_{1} \tau_{4} \tau_{5}+\tau_{3} \tau_{4} \tau_{5}\right)+\beta_{4} \tau_{1} \tau_{2} \tau_{3}\right] s^{3} \\
& +\left[\left(\beta_{6}+\beta_{5}\right)\left(\tau_{1} \tau_{2}+\tau_{1} \tau_{4}+\tau_{3} \tau_{4}\right)+\beta_{3} \tau_{1} \tau_{2}\right] s^{2} \\
& +\left[\beta_{6}\left(\tau_{1}+\tau_{3}+\tau_{5}\right)+\beta_{4}\left(\tau_{1}+\tau_{3}\right)+\beta_{2} \tau_{1}\right] s \\
& +\left(\beta_{6}+\beta_{5}+\beta_{3}+\beta_{1}\right) \\
& \beta_{6}=A_{5} / B_{5} \\
& \beta_{5}=A_{4} / B_{4}-\beta_{6} \\
& \beta_{4}=\left(A_{3}-\beta_{6} B_{3}\right) / \tau_{1} \tau_{2} \tau_{3} \\
& \beta_{3}=\left[A_{2}-\left(\beta_{6}+\beta_{5}\right) B_{2}-\beta_{5} B_{2}\right] / \tau_{1} \tau_{2} \\
& \beta_{2}=\left[A_{1}-\beta_{6} B_{1}-\beta_{4}\left(\tau_{1}+\tau_{3}\right)\right] / \tau_{1} \\
& \beta_{1}=A_{0}-\left(\beta_{6}+\beta_{5}+\beta_{3}\right) \\
& n=6: N(s)=\beta_{7} \tau_{1} \tau_{2} \tau_{3} \tau_{4} \tau_{5} \tau_{6} s^{6} \\
& +\left(\beta_{7}+\beta_{6}\right) \tau_{1} \tau_{2} \tau_{3} \tau_{4} \tau_{5} s^{5} \\
& +\left[\beta _ { 7 } \left(\tau_{1} \tau_{2} \tau_{3} \tau_{4}+\tau_{1} \tau_{2} \tau_{3} \tau_{6}+\tau_{1} \tau_{2} \tau_{5} \tau_{6}+\tau_{1} \tau_{4} \tau_{5} \tau_{6}\right.\right. \\
& \left.\left.+\tau_{3} \tau_{4} \tau_{5} \tau_{6}\right)+\beta_{5} \tau_{1} \tau_{2} \tau_{3} \tau_{4}\right] s^{4} \\
& +\left[\left(\beta_{7}+\beta_{6}\right)\left(\tau_{1} \tau_{2} \tau_{3}+\tau_{1} \tau_{2} \tau_{5}+\tau_{1} \tau_{4} \tau_{5}+\tau_{3} \tau_{4} \tau_{5}\right)\right. \\
& \left.+\beta_{4} \tau_{1} \tau_{2} \tau_{3}\right] s^{3} \\
& +\left[\left(\beta_{7}+\beta_{5}\right)\left(\tau_{1} \tau_{2}+\tau_{1} \tau_{4}+\tau_{3} \tau_{4}\right)\right.
\end{aligned}
$$

$$
\begin{aligned}
H(s) & =V_{\text {output }} / V_{\text {input }} \\
& =\alpha_{0}+\sum_{j=1}^{n} \alpha_{j} H_{j}(s) \\
H(s) & =\alpha_{0}+\frac{1}{\tau_{1} s N_{H 1}(s)+N_{H 2}(s)} \sum_{j=1}^{n} \alpha_{j} N_{H j}(s) .
\end{aligned}
$$

Note that $N_{H j}(s)$ can be obtained iteratively as shown in (3) and given in Section II-A for $n=3-6$.

Using (3) and (42), we can formulate the numerators $N(s)$ of the transfer functions and the corresponding design formulas of 
summation parameters $\alpha_{j}$ when realizing the general function in (27) for $n=3-6$, given as

$$
\begin{aligned}
& n=3: N(s)=\alpha_{0} \tau_{1} \tau_{2} \tau_{3} s^{3}+\left(\alpha_{0} \tau_{1} \tau_{2}+\alpha_{1} \tau_{2} \tau_{3}\right) s^{2} \\
& +\left[\alpha_{0}\left(\tau_{1}+\tau_{3}\right)+\alpha_{1} \tau_{2}+\alpha_{2} \tau_{3}\right] s \\
& +\left(\alpha_{0}+\alpha_{1}+\alpha_{2}+\alpha_{3}\right) \\
& \alpha_{0}=A_{3} / B_{3} \\
& \alpha_{1}=\left(A_{2}-\alpha_{0} B_{2}\right) / \tau_{2} \tau_{3} \\
& \alpha_{2}=\left(A_{1}-\alpha_{0} B_{1}-\alpha_{1} \tau_{2}\right) / \tau_{3} \\
& \alpha_{3}=A_{0}-\left(\alpha_{0}+\alpha_{1}+\alpha_{2}\right) \\
& n=4: N(s)=\alpha_{0} \tau_{1} \tau_{2} \tau_{3} \tau_{4} s^{4} \\
& +\left(\alpha_{0} \tau_{1} \tau_{2} \tau_{3}+\alpha_{1} \tau_{2} \tau_{3} \tau_{4}\right) s^{3} \\
& \left.+\left[\alpha_{0}\left(\tau_{1} \tau_{2}+\tau_{1} \tau_{4}+\tau_{3} \tau_{4}\right)+\alpha_{1} \tau_{2} \tau_{3}+\alpha_{2} \tau_{3} \tau_{4}\right)\right] s^{2} \\
& +\left[\alpha_{0}\left(\tau_{1}+\tau_{3}\right)+\alpha_{1}\left(\tau_{2}+\tau_{4}\right)+\alpha_{2} \tau_{3}+\alpha_{3} \tau_{4}\right] s \\
& +\left(\alpha_{0}+\alpha_{1}+\alpha_{2}+\alpha_{3}+\alpha_{4}\right) \\
& \alpha_{0}=A_{4} / B_{4} \\
& \alpha_{1}=\left(A_{3}-\alpha_{0} B_{3}\right) / \tau_{2} \tau_{3} \tau_{4} \\
& \alpha_{2}=\left(A_{2}-\alpha_{0} B_{2}-\alpha_{1} \tau_{2} \tau_{3}\right) / \tau_{3} \tau_{4} \\
& \alpha_{3}=\left[A_{1}-\alpha_{0} B_{1}-\alpha_{1}\left(\tau_{2}+\tau_{4}\right)-\alpha_{2} \tau_{3}\right] / \tau_{4} \\
& \alpha_{4}=A_{0}-\left(\alpha_{0}+\alpha_{1}+\alpha_{2}+\alpha_{3}\right) \\
& n=5: N(s)=\alpha_{0} \tau_{1} \tau_{2} \tau_{3} \tau_{4} \tau_{5} s^{5} \\
& +\left(\alpha_{0} \tau_{1} \tau_{2} \tau_{3} \tau_{4}+\alpha_{1} \tau_{2} \tau_{3} \tau_{4} \tau_{5}\right) s^{4} \\
& +\left[\alpha_{0}\left(\tau_{1} \tau_{2} \tau_{3}+\tau_{1} \tau_{2} \tau_{5}+\tau_{1} \tau_{4} \tau_{5}+\tau_{3} \tau_{4} \tau_{5}\right)\right. \\
& \left.+\alpha_{1} \tau_{2} \tau_{3} \tau_{4}+\alpha_{2} \tau_{3} \tau_{4} \tau_{5}\right] s^{3} \\
& +\left[\alpha_{0}\left(\tau_{1} \tau_{2}+\tau_{1} \tau_{4}+\tau_{3} \tau_{4}\right)+\alpha_{1}\left(\tau_{2} \tau_{3}+\tau_{2} \tau_{5}+\tau_{4} \tau_{5}\right)\right. \\
& \left.+\alpha_{2} \tau_{3} \tau_{4}+\alpha_{3} \tau_{4} \tau_{5}\right] s^{2} \\
& +\left[\alpha_{0}\left(\tau_{1}+\tau_{3}+\tau_{5}\right)+\alpha_{1}\left(\tau_{2}+\tau_{4}\right)+\alpha_{2}\left(\tau_{3}+\tau_{5}\right)\right. \\
& \left.+\alpha_{3} \tau_{4}+\alpha_{4} \tau_{5}\right] s+ \\
& \left(\alpha_{0}+\alpha_{1}+\alpha_{2}+\alpha_{3}+\alpha_{4}+\alpha_{5}\right) \\
& \alpha_{0}=A_{5} / B_{5} \\
& \alpha_{1}=\left(A_{4}-\alpha_{0} B_{4}\right) / \tau_{2} \tau_{3} \tau_{4} \tau_{5} \\
& \alpha_{2}=\left(A_{3}-\alpha_{0} B_{3}-\alpha_{1} \tau_{2} \tau_{3} \tau_{4}\right) / \tau_{3} \tau_{4} \tau_{5} \\
& \alpha_{3}=\left[A_{2}-\alpha_{0} B_{2}-\alpha_{1}\left(\tau_{2} \tau_{3}+\tau_{2} \tau_{5}+\tau_{4} \tau_{5}\right)\right. \\
& \left.-\alpha_{2} \tau_{3} \tau_{4}\right] / \tau_{4} \tau_{5} \\
& \alpha_{4}=\left[A_{1}-\alpha_{0} B_{1}-\alpha_{1}\left(\tau_{2}+\tau_{4}\right)-\alpha_{2}\left(\tau_{3}+\tau_{5}\right)\right. \\
& \left.-\alpha_{3} \tau_{4}\right] / \tau_{5} \\
& \alpha_{5}=A_{0}-\left(\alpha_{0}+\alpha_{1}+\alpha_{2}+\alpha_{3}+\alpha_{4}\right) \\
& n=6: N(s)=\alpha_{0} \tau_{1} \tau_{2} \tau_{3} \tau_{4} \tau_{5} \tau_{6} s^{6} \\
& +\left(\alpha_{0} \tau_{1} \tau_{2} \tau_{3} \tau_{4} \tau_{5}+\alpha_{1} \tau_{2} \tau_{3} \tau_{4} \tau_{5} \tau_{6}\right) s^{5} \\
& +\left[\alpha _ { 0 } \left(\tau_{1} \tau_{2} \tau_{3} \tau_{4}+\tau_{1} \tau_{2} \tau_{3} \tau_{6}+\tau_{1} \tau_{2} \tau_{5} \tau_{6}\right.\right. \\
& \left.+\tau_{1} \tau_{4} \tau_{5} \tau_{6}+\tau_{3} \tau_{4} \tau_{5} \tau_{6}\right) \\
& \left.+\alpha_{1} \tau_{2} \tau_{3} \tau_{4} \tau_{5}+\alpha_{2} \tau_{3} \tau_{4} \tau_{5} \tau_{6}\right] s^{4} \\
& +\left[\alpha_{0}\left(\tau_{1} \tau_{2} \tau_{3}+\tau_{1} \tau_{2} \tau_{5}+\tau_{1} \tau_{4} \tau_{5}+\tau_{3} \tau_{4} \tau_{5}\right)\right. \\
& +\alpha_{1}\left(\tau_{2} \tau_{3} \tau_{4}+\tau_{2} \tau_{3} \tau_{6}+\tau_{2} \tau_{5} \tau_{6}+\tau_{4} \tau_{5} \tau_{6}\right) \\
& \left.+\alpha_{2} \tau_{3} \tau_{4} \tau_{5}+\alpha_{3} \tau_{4} \tau_{5} \tau_{6}\right] s^{3} \\
& +\left[\alpha_{0}\left(\tau_{1} \tau_{2}+\tau_{1} \tau_{4}+\tau_{1} \tau_{6}+\tau_{3} \tau_{4}+\tau_{3} \tau_{6}+\tau_{5} \tau_{6}\right)\right. \\
& +\alpha_{1}\left(\tau_{2} \tau_{3}+\tau_{2} \tau_{5}+\tau_{4} \tau_{5}\right)+\alpha_{2}\left(\tau_{3} \tau_{4}+\tau_{3} \tau_{6}+\tau_{5} \tau_{6}\right) \\
& \left.+\alpha_{3} \tau_{4} \tau_{5}+\alpha_{4} \tau_{5} \tau_{6}\right] s^{2}
\end{aligned}
$$
and iterive and useful for quick design of LF-based all-pole and arbitrary transmission zero filters without the need for reformulation.

\section{REFERENCES}

[1] R. Schaumann, M. S. Ghausi, and K. R. Laker, Design of Analog Filters: Passive, Active-RC, and Switched Capacitor. Englewood Cliffs, NJ: Prentice-Hall, 1990.

[2] T. Deliyannis, Y. Sun, and J. K. Fidler, Continuous-Time Active Filter Design. Boca Raton, FL: CRC, 1999.

[3] R. Nawrocki, "Electronically tunable all-pole low-pass leapfrog ladder filter with operational transconductance amplifier," Int. J. Electron., vol. 62, pp. 667-672, 1987.

[4] A. C. M. de Queiroz, L. P. Caloba, and E. Sanchez-Sinencio, "Signal flow graph OTA-C integrated filters," in Proc. IEEE ISCAS, 1988, pp. 2165-68.

[5] R. Schaumann, "Simulating lossless ladders with transconductance-C circuits," IEEE Trans. Circuits Syst. II, Analog Digit. Signal Process. vol. 45, no. 3, pp. 407-410, Mar. 1998.

[6] Y. Sun and J. K. Fidler, "Structure generation and design of multiple loop feedback OTA-grounded capacitor filters," IEEE Trans. Circuits Syst. I, Fundam. Theory Appl., vol. 44, no. 1, pp. 1-11, Jan. 1997.

[7] _ "Current-mode multiple-loop feedback filters using dual-output OTAs and grounded capacitors," Int. J. Circuit Theory Appl., vol. 25, pp. 69-80, 1997.

[8] H. W. Su and Y. Sun, "Performance analysis and comparison of multiple loop feedback OTA-C filters," Int. J. Circuits, Syst. and Comput., vol. 14 , no. $4,2005$.

[9] H. W. Su and Y. Sun, "A CMOS $100 \mathrm{MHz}$ continuous-time seventhorder $0.05^{\circ}$ equiripple linear phase leapfrog multiple loop feedback $G_{\mathrm{m}}-C$ filter," in Proc. IEEE ISCAS, May 2002, vol. 2, pp. 17-20.

[10] Y. Sun and J. K. Fidler, "Current-mode OTA-C realisation of arbitrary filter characteristics," Electron. Lett., vol. 32, no. 13, pp. 1181-82, 1996.

[11] C. A. Barbargires, "Explicit design of general high-order FLF OTA-C filters," Electron. Lett., vol. 35, no. 16, pp. 1289-1290, 1999.

[12] Y. Sun and J. K. Fidler, "OTA-C realization of general high-order transfer functions," Electron. Lett., vol. 29, no. 12, pp. 1057-58, 1993.

[13] _ _ "Synthesis and performance analysis of universal minimum component integrator-based IFLF OTA-grounded capacitor filter," Proc. Inst. Elect. Eng. CDS, vol. 143, no. 2, pp. 107-114, 1996.

[14] D. H. Chiang and R. Schaumann, "Design of a CMOS fully-differential continuous-time tenth-order filter based on IFLF topology," in Proc. IEEE ISCAS, 1998, vol. 1, pp. 123-126. 